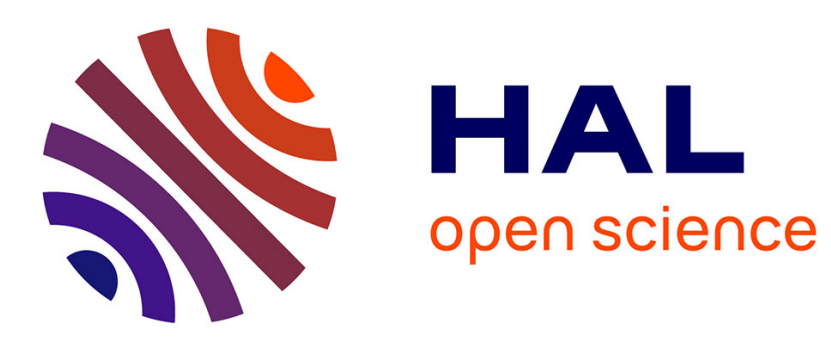

\title{
Models and Algorithms for Intermodal Transportation and Equipment Selection
}

\author{
Christina Burt, Jakob Puchinger
}

\section{To cite this version:}

Christina Burt, Jakob Puchinger. Models and Algorithms for Intermodal Transportation and Equipment Selection. International Conference on Operations Research (OR 2011), Aug 2011, Zurich, Switzerland. 10.1007/978-3-642-29210-1_49 . hal-01307975

\section{HAL Id: hal-01307975 \\ https://hal.inria.fr/hal-01307975}

Submitted on 27 Apr 2016

HAL is a multi-disciplinary open access archive for the deposit and dissemination of scientific research documents, whether they are published or not. The documents may come from teaching and research institutions in France or abroad, or from public or private research centers.
L'archive ouverte pluridisciplinaire HAL, est destinée au dépôt et à la diffusion de documents scientifiques de niveau recherche, publiés ou non, émanant des établissements d'enseignement et de recherche français ou étrangers, des laboratoires publics ou privés. 


\title{
Models and Algorithms for Intermodal Transportation and Equipment Selection
}

\author{
Christina Burt and Jakob Puchinger
}

\begin{abstract}
We study a cement transportation problem with asset-management (equipment selection) for strategic planning scenarios involving multiple forms of transportation and network expansion. A deterministic mixed integer programming approach is difficult due to the underlying capacitated multi-commodity network flow model and the need to consider a time-space network to ensure operational feasibility. Considering uncertain aspects is also difficult but important. In this paper we propose three approaches: solve a deterministic mixed integer program optimally; solve stochastic programs to obtain robust bounds on the solution; and, study alternative solutions to obtain their optimal cost vectors using inverse programming.
\end{abstract}

\section{Introduction}

We consider a strategic network design problem with asset management, inspired by the requirements of a leading European construction group currently delivering cement in a truck transportation system. The company is considering opportunities to expand the network to incorporate intermodal transportation by adding rail and/or barge links. Additional factories are also considered and as such, the production schedule is not known.

Given a set of cement orders, we wish to select transportation equipment and related arcs; select services and associated schedules; and build flow paths through the network such that a production schedule at each factory can also be generated. We aim to achieve the optimum strategic network design at minimum cost given that certain aspects of costing are uncertain. Since we permit multi-commodity flows to satisfy orders, and the selection of services and equipment requires a fixed charge in the objective function, this problem is strongly related to and is likely to be at least as difficult as the fixed charge capacitated multi-commodity flow network problemknown to be $\mathscr{N} \mathscr{P}$-hard [7]. Adding to this difficulty is the scale of the time-space network.

AIT Austrian Institute of Technology, Mobility Department, Vienna

e-mail: \{christina.burt.fl|jakob.puchinger\}@ait.ac.at 
Similar problems have been modelled as multi-commodity flow mixed integer program on time-space networks [2]; however, it has been long known that the bound provided by the $L P$ relaxation is weak [4]. Worse, the time-space network can lead to an inhibiting number of variables, such as in [11]. Since there are uncertain aspects to our problem — notably costs and demands—a stochastic programming approach is desirable. In the closely related context of service network design, a two-stage stochastic programming model [10] has been succesfully used. Preprocessing (see [5]), decomposition (see [3,9]), and cutting planes (see [4-6]) have helped improve the tractability of the underlying network model. However, in genuine applications, such as [2], this has been insufficient to produce a thorough stability analysis.

We propose a portfolio of solution approaches that generate a complementary set of future options. In this paper we propose three approaches: solve a deterministic mixed integer program optimally (Section 2); generate solutions to a simplified stochastic program (Section 3); and study the expected solutions to obtain their optimal cost vectors (Section 4). We briefly outline future challenges in Section 5.

\section{Model}

We define the network on a graph $G=(\mathscr{N}, \mathscr{A})$ where $\mathscr{N}$ is the set of nodes and $\mathscr{A}$ is the set of paths connecting each pair. Each arc is indexed by the nodes, $i$ and $j$, and equipment, $e$, that moves between, giving the tuple $a=(i, j, e)$. We model the problem as a multi-commodity network flow mixed integer program, where the commodities represent the capability to satisfy orders from more than one factory.

The flow variables, $\mathbf{x}_{a, k}^{t}$, capture the flow of cement on $\operatorname{arc} a$ at the start of period $t$ for commodity $k$. The equipment index is important in ensuring that the commodity is not split once it has left the factory in one package (since it is not practical to split cement packaging). The service variables, $\mathbf{y}_{a}^{t}$, indicate whether a service by equipment $e(\in a)$ runs on $\operatorname{arc} a$ at the start of period $t$. These variables can be used to also indicate the time of purchase of a particular machine by comparing the existence of services provided in period $t$ with the existence of services provided in possible previous time periods, $t-t\left(a^{\prime}\right)$, where $t\left(a^{\prime}\right)$ is the service time along a preceding arc $a^{\prime}$ (see Figure ??). Salvage time is indicated for each equipment by binary variable $\mathbf{s}^{t, e}$.

The objective function minimises the cost of owning $\left(f^{e}\right)$ and operating $\left(v^{a}\right)$ the equipment. All costs are discounted back to the present using a discount factor, $d^{t}=\frac{1}{(1+I)^{t}}$, for some interest rate $I$. Transshipment nodes may incur a fixed fee when the flow changes mode of transport, as indicated by binary variable $\mathbf{z}_{k}^{t}$.

The movement of cement through the network is modelled with flow constraints: demand constraints for each order, $\kappa$, with associated due date, $\tau$, implied by the order (Constraint 1); node balancing for interior nodes (Constraint 2) and the demand nodes (Constraint 3) - this captures the ability of equipment to drop off partial orders; and, capacity constraints on the production nodes, $\mathscr{N}^{O}$ (Constraint 4). 
Since the production schedule is not known a priori, the latter is an inequality. Decision variables are linked by Constraint (5), where $c_{e}$ is the maximum capacity of a provided service. The disaggregated right hand side prevents commodity splitting across equipment within an arc. Special-ordered set constraints (type 1) prevent flow splitting across arcs (Constraint 6) and across equipment (Constraint 7).

$$
\begin{array}{r}
\min \sum_{e} f^{e} \sum_{a} \mathbf{y}_{a}^{0}+\sum_{i, e, t>0} d^{t} f_{\pi}^{e}\left[\sum_{a=(i, j, e)} \mathbf{y}_{a}^{t}-\sum_{a^{\prime}=\left(i^{\prime}, i, e\right)} \mathbf{y}_{a^{\prime}}^{t-t\left(a^{\prime}\right)}+\mathbf{s}^{t, e}\right]+\sum_{a, t} d^{t} v_{y}^{a} \mathbf{y}_{a}^{t} \\
+\sum_{a, k, t} d^{t} v_{x}^{a} \mathbf{x}_{a, k}^{t}+\sum_{k, t} d^{t} f^{\tau} \mathbf{z}_{k}^{t}-\sum_{e, t} d^{t} d_{2}^{t} f^{e} \mathbf{s}^{t, e}
\end{array}
$$

subject to

$$
\begin{aligned}
& \sum_{\substack{\{a=(i, j, e) \mid j \in \kappa\}, k, \tau^{\kappa}-\tau^{0}<t<\tau^{\kappa}}} \mathbf{x}_{a, k}^{t-t(a)} \geq \sum_{\substack{\left\{a=\left(j, j j^{\prime}, e\right) \mid j \in \kappa\right\}, k, \tau^{\kappa}-\tau^{0}<t<\tau^{\kappa}}} \mathbf{x}_{a, k}^{t}+D^{\kappa} \quad \forall \kappa, \\
& \sum_{a=(i, j, e)} \mathbf{x}_{a, k}^{t-t(a)}=\sum_{a^{\prime}=\left(j, j^{\prime}, e\right)} \mathbf{x}_{a^{\prime}, k}^{t} \quad \forall j \in \mathscr{N} \backslash\left\{\mathscr{N}^{O} \cup \mathscr{N}^{D}\right\}, k, t, \\
& \sum_{a=(i, j, e), k, t} \mathbf{x}_{a, k}^{t}=\sum_{a^{\prime}=\left(j, j^{\prime}, e\right), k, t} \mathbf{x}_{a^{\prime}, k}^{t}+\sum_{\kappa \mid j \in \kappa} D^{\kappa} \quad \forall j \in \mathscr{N}^{D} \\
& \sum_{a=(i, j, e), k} \mathbf{x}_{a, k}^{t} \leq P_{i}^{t} \quad \forall i \in \mathscr{N}^{O}, t, \\
& \sum_{k} \mathbf{x}_{a, k}^{t} \leq c_{e} \mathbf{y}_{a}^{t} \quad \forall a, t \\
& \operatorname{SOS} 1\left(\mathbf{x}_{a, k}^{t} \mid(i, j) \in \mathscr{A}\right) \quad \forall e, k, t \\
& \operatorname{SOS} 1\left(\mathbf{x}_{a, k}^{t} \mid e \in \mathscr{E}\right) \quad \forall(i, j), k, t \\
& \sum_{a} \mathbf{y}_{a}^{t} \leq 1 \quad \forall e, t, \\
& \mathbf{x}_{a, k}^{t-t(a)}+\sum_{a^{\prime}=\left(j, j^{\prime}, e^{\prime}\right) \mid e^{\prime} \in \mathscr{E} \backslash e} \mathbf{x}_{a^{\prime}, k}^{t}-\sum_{a^{\prime}=\left(j, j^{\prime}, e^{\prime}\right) \mid e^{\prime} \in \mathscr{E}} \mathbf{x}_{a^{\prime}, k}^{t} \leq M \mathbf{z}_{k}^{t} \quad \forall a, k, t \in[t(a), T], \\
& \mathbf{y}_{a}^{t-t(a)} \leq \sum_{a^{\prime} \in\left(j, j^{\prime}\right)} \mathbf{y}_{a^{\prime}}^{t}+s^{t-1, e} \quad \forall a, t \in[t(a), T], \\
& \sum_{t}\left(\sum_{a=(i, j, e)} \mathbf{y}_{a}^{t}-\sum_{a^{\prime}=\left(i^{\prime}, i, e\right)} \mathbf{y}_{a^{\prime}}^{t-t\left(a^{\prime}\right)}\right) \leq 1 \quad \forall e \\
& \mathbf{s}^{t, e} \leq \sum_{a, h \leq t} \mathbf{y}_{a}^{h} \quad \forall e, t, \\
& \sum_{t} \mathbf{s}^{t, e} \leq 1 \quad \forall e, \\
& \sum_{a, h>t} \mathbf{y}_{a}^{h} \leq\left(1-\mathbf{s}^{t, e}\right) M \quad \forall e, t, \\
& \mathbf{y}_{a}^{t} \in\{0,1\} \quad \forall a, t, \\
& \mathbf{z}_{k}^{t} \in\{0,1\} \quad \forall t, k, \\
& \mathbf{s}^{t, e} \in\{0,1\} \quad \forall t, e, \\
& \mathbf{x}_{a, k}^{t} \in \mathscr{R}^{+} \quad \forall a, k, t .
\end{aligned}
$$




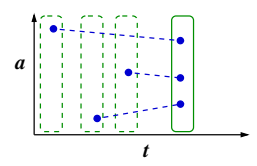

Fig. 1 Purchases are determined by looking at all arc service variables in one time period and comparing it with service variables in the immediately preceding possible time period.

SOS 1 constraints allow at most one variable in the set to be non-zero. Since the service variables are binary, we use a knapsack constraint to prevent equipment splitting across arcs (Constraint 8). The time of transshipment is captured in Constraint (9). Consistency in the time-space network is provided for service by the restriction (10). We restrict purchasing in Constraint (11), using a similar argument as illustrated in Figure ??. Finally, we ensure consistency in the salvage variables (Constraints (12)-(14)). We reduce the domains of the constraints by considering edge conditions, and exclude variables never appearing in the optimal solution.

The literature suggests that solving the full, deterministic mixed-binary model without simplification will be difficult. Both preprocessing and decomposition approaches are required to achieve tractability. Obvious preprocessing includes disjoint sets, precedence, cycles and covering. However, we need to be mindful that the preprocessing doesn't break the strong structure provided for decomposition by the time-space network. Therefore much analysis is needed for this approachespecially if the final implementation is to avoid the use of commercial solvers.

\section{Stochastic Programming Approach}

We propose a two-stage stochastic programming approach. The equipment and salvage variables are fixed in the first stage. In the second stage, some random events $\omega \in \Omega$ are realised and determine the random vector $\chi$ consisting of: the operating cost coefficients $v^{a}(\omega)$; and, the demand $D^{\kappa}$. The recourse action then consists of decisions about the service and flow variables. Assuming a fixed number of realisations/scenarios for $\chi$ the stochastic program can be defined in its extensive form. Furthermore, all binary variables are fixed in the first stage. Therefore the proposed stochastic program can be solved using a standard algorithm such as the $L$-shaped method. The model defined in Section 2 includes the possibility of outsourcing transport at a high cost, ensuring the feasibility of the second stage.

Since the linear program of the second stage can be solved efficiently, it remains to be shown how to fix the first stage variables. On the one hand this could be done by exatly solving the first stage at every iteration of the L-shaped method using a mixed integer programming solver. On the other hand for problem instances of realistic size the use of heuristics might become necessary [8]. In our case, first stage decisions could be taken by first solving the flow problem and then allowing 
the flow to imply the services required for each scenario. Then the decision about the equipment and factories can be taken based on the required services.

\section{Scenario-scaping Heuristic}

An alternative approach is to develop an understanding of when alternate solutions would become optimal. To achieve this, we propose a heuristic to approximate the cost vector that would make an initial feasible solution optimal. The proposed heuristic itself is outlined in Algorithm 1. We will begin with a good feasible solution proposed by the industry partner. We will then use inverse programming (as proposed in [1]) under the $L_{1}$ norm to find the nearest cost vector that makes the feasible solution optimal. This is a type of reverse stochastic programming: instead of determining the best decision under uncertainty, we provide the conditions under which each decision will be best. This is particularly useful for decision-making if the industry partner has a way of influencing the cost vector or has undisclosed cost information.

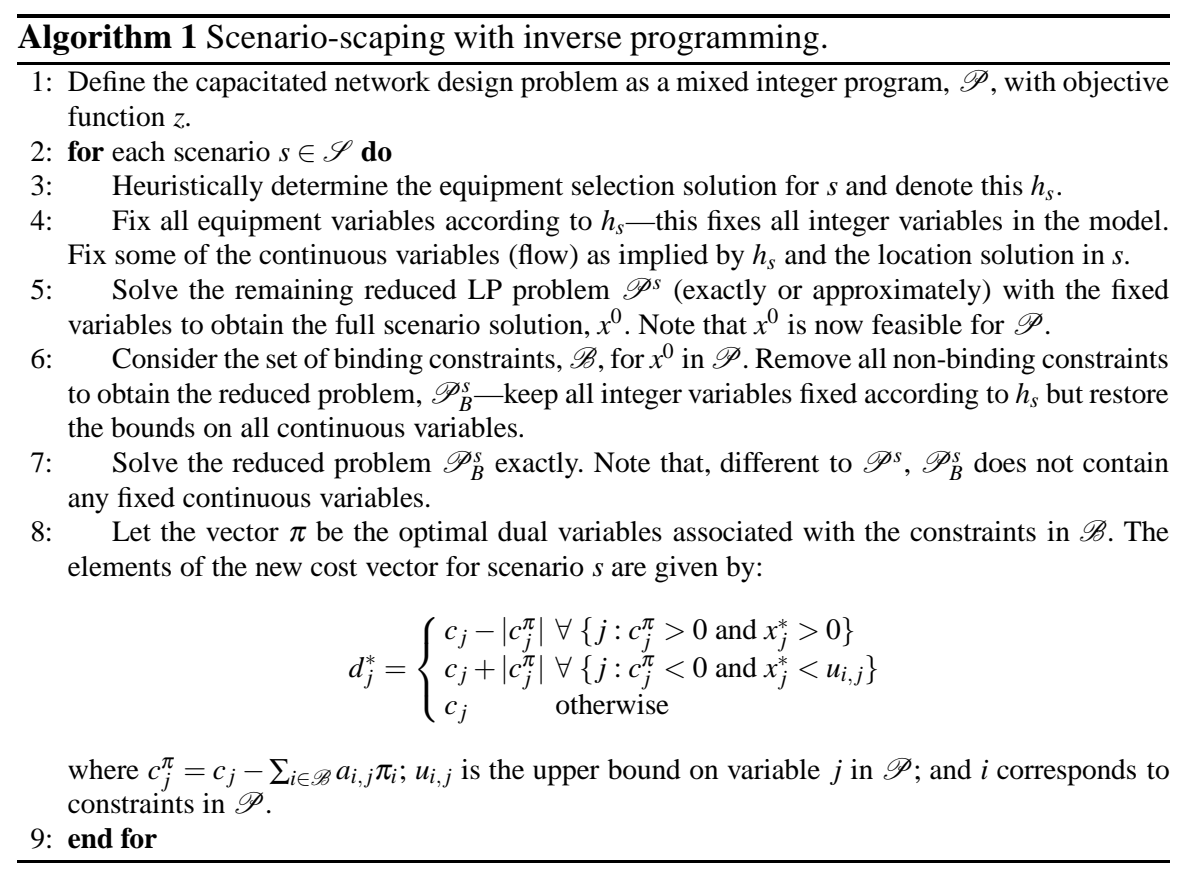

We obtain the initial feasible solution $x^{0}$ by first fixing many (integer) variables when we define the scenario and solving the remaining reduced mixed integer program approximately. The SOS constraints create a non-convex feasible region. To combat this we propagate the SOS constraints to obtain the finite set of convex $L P$ 's 
that comprise the feasible region-since these $L P$ 's are already significantly reduced it is trivial to enumerate through the set to obtain the optimal solution.

\section{Conclusions and Discussion}

In this paper we have studied a difficult network design problem and proposed several approaches, which combine to provide supporting information in a holistic manner. This is important given the cost of making these decisions and the uncertainty involved in the cost vector. In this way, we meet our goal of obtaining quality solutions while taking into account that the genuine optimal solution is influenced by events we cannot foresee. The complementary solutions we provide will empower our industry partner to make good and near-optimal decisions even if they need to withhold (or cannot quantify) confidential data.

Acknowledgements This work is part of the project I2Bau, partially funded by the Austrian Federal Ministry for Transport, Innovation and Technology (BMVIT) within the strategic programme I2VSplus under grant 826188 .

\section{References}

1. R. K. Ahuja And J. B. Orlin, Inverse optimization, Operations Research, 49 (2001), pp. 771-783.

2. J. Anderson, T. G. Crainic, And M. Christiansen, Service network design with asset management: Formulations and comparative analyses, Transportation Research, 17 (2009), pp. 197-207.

3. C. Barnhart, N. Krishnan, D. Kim, AND K. WARE, Network design for express shipment delivery, Computational Optimization and Applications, 21 (2002), pp. 239-262.

4. D. BIENSTOCK AND G. MURATORE, Strong inequalities for capacitated survivable network design problems, Mathematical Programming, 89 (2000), pp. 127-147.

5. N. Boland, M. KRishnamoorthy, A. ERnst, AND J. Ebery, Preprocessing and cutting for multiple allocation hub location problems, European Journal of Operational Research, 155 (2004), pp. 638-653.

6. J.-F. CORDEAU, M. Iori, G. Laporte, AND J. J. S. GonZalez, A branch-and-cut algorithm for the pickup and delivery traveling salesman problem with lifo loading, tech. report, CIRRELT, 2007.

7. B. Gendron, T. Crainic, And A. Frangioni, Telecommunications Network Planning, Kluwer Academic, 1998, ch. Multicommodity capacitated network design.

8. A. Hoff, A.-G. LiUm, A. LøKKETANGEN, AND T. CRAINIC, A metaheuristic for stochastic service network design, Journal of Heuristics, 16 (2010), pp. 653-679.

9. S. IRNICH, Netzwerk-Design für zweistufige Transportsysteme und ein Branch-and-PriceVerfahren für das gemischte Direkt- und Hubflugproblem, $\mathrm{PhD}$ thesis, Lehrstuhl für Unternehmensforschung, Rheinisch-Westfälische Technische Hochschule, 2002.

10. A.-G. LiUm, T. G. CRAinic, AND S. W. WAllace, A study of demand stochasticity in service network design, TRANSPORTATION SCIENCE, 43 (2009), pp. 144-157.

11. Y. ZHANG, Advances in LTL load plan design, $\mathrm{PhD}$ thesis, Georgia Institute of Technology, 2010. 